\title{
Early discontinuation of empirical antibiotic treatment in neutropenic patients with acute myeloid leukaemia and high-risk myelodysplastic syndrome
}

Floor Annabel Niessen ( $\nabla$ f.a.niessen@umcutrecht.nl )

Universitair Medisch Centrum Utrecht https://orcid.org/0000-0001-6586-9776

Maaike S. M. van Mourik

Universitair Medisch Centrum Utrecht

Anke H. W. Bruns

Universitair Medisch Centrum Utrecht

Reinier A. P. Raijmakers

Universitair Medisch Centrum Utrecht

Mark C.H. de Groot

Universitair Medisch Centrum Utrecht

Tjomme van der Bruggen

Universitair Medisch Centrum Utrecht

\section{Research}

Keywords: Carbapenems, febrile neutropenia, acute myeloid leukaemia, antibiotic stewardship

Posted Date: February 6th, 2020

DOI: https://doi.org/10.21203/rs.2.17037/v2

License: (c) (1) This work is licensed under a Creative Commons Attribution 4.0 International License. Read Full License

Version of Record: A version of this preprint was published at Antimicrobial Resistance and Infection Control on May 27th, 2020. See the published version at https://doi.org/10.1186/s13756-020-00729-2. 


\section{Abstract}

Introduction: Current guidelines advocate empirical antibiotic treatment (EAT) in haematological patients with febrile neutropenia. However, the optimal duration of EAT is unknown. In 2011, we have introduced a protocol, promoting discontinuation of carbapenems as EAT after three days in most patients and discouraging the standard use of vancomycin as EAT.[NF1] This study assesses the effect of this protocol on carbapenem and vancomycine use in high risk haematological patients and its safety.

Methods: A retrospective before-after study was performed comparing a cohort from 2007 to 2011 (period I, before restrictive EAT use) with a cohort from 2011-2014 (period II, restrictive EAT use). Neutropenic episodes related to chemotherapy or stem cell transplantation (SCT) in patients with acute myeloid leukaemia (AML) or high-risk myelodysplastic syndrome (MDS) were analysed. The primary outcome was the use of carbapenems and vancomycin as EAT during neutropenia, expressed as days of therapy (DOT)/100 neutropenic days and analysed with interrupted time series (ITS). Also the use of other antibiotics was analysed to evaluate the overall antibiotic use[NF2] . Safety measurements included 30-day mortality, ICU admittance within 30 days after start of EAT and blood cultures positive for microorganisms sensitive to a carbapenem.

Results: 362 neutropenic episodes with a median duration of 18 days were analysed, involving 201 patients. ITS analysis showed decreased carbapenem use with a step change of $16.1 \mathrm{DOT} / 100$ neutropenic days ( $\mathrm{Cl}-26.73$ to -1.41$)$ and an overall reduction of $21.6 \%$ (8.7 DOT/100 neutropenic days). [NF3] Vancomycin use decreased with a step change of 13.7 DOT/100 neutropenic days ( $95 \% \mathrm{Cl}-23.66$ to -2.90 ) and an overall reduction of $54.7 \%$ (14.6 DOT/100 neutropenic days). [NF4] There were no striking differences in other therapeutically used broad-spectrum antibiotics. No deaths were directly related to early discontinuation of EAT, also no notable difference in ICU-admission ( $n=9$ in period $\mathrm{I}, \mathrm{n}=9$ in period II) and positive blood cultures ( $\mathrm{n}=4$ in period $\mathrm{I}, \mathrm{n}=2$ in period II) [NF5] was detected.

Conclusion: The introduction of a protocol promoting restrictive use of EAT resulted in reduction of carbapenem and vancomycin use and appears to be safe in AML or high-risk MDS patients with febrile neutropenia during chemotherapy or SCT.

\section{Introduction}

Patients with acute myeloid leukaemia (AML) or high-risk myelodysplastic syndrome (MDS) are treated with intensive chemotherapy and, if indicated, an allogenic stem cell transplantation (SCT). This treatment results in periods of neutropenia with mucositis, making patients vulnerable to severe infections. Due to the mucosal barrier injury, microorganisms from the gastrointestinal tract can translocate to the bloodstream, including Gram-negative bacteria, viridans streptococci and Candida spp.. Therefore, patients receive antimicrobial prophylaxis during the period of neutropenia. Despite prophylaxis, more than $50 \%$ of these patients become febrile $(1,2)$. 
When a patient develops fever during neutropenia, broad spectrum empirical antibiotic treatment (EAT) is started immediately to rapidly and adequately treat a bacterial infection. Empirical antibiotic options all include coverage of Pseudomonas aeruginosa. Commonly used EAT regimes are a carbapenem, piperacillin/tazobactam, ceftazidime or cefepime with or without an aminoglycoside $(1,3)$.

Current guidelines advocate to continue EAT until the patient has been afebrile for $\geq 48$ hours (3) or even as long as the duration of neutropenia (1). However, fever during neutropenia does not necessarily have a bacterial etiology. Viral and fungal infections are also frequently encountered. In addition, in $30-50 \%$ of cases no causative pathogen can be identified (4). The haematological malignancy, administration of blood products, or mucositis itself might also cause fever $(5,6)$. Current protocols advocating continuous EAT in neutropenic fever may therefore lead to overtreatment with broad-spectrum antibiotics and associated risks of side effects and antibiotic resistance. The broad spectrum antibiotics used as EAT are listed by the World Health Organization (WHO) as critically important antimicrobials for human medicine (Watch category of the AWaRe classification) and are considered key targets of stewardship programs and monitoring (7).

Recent studies suggest that protocols with a more restrictive use of EAT can be safely implemented $(8,9)$. A prospective study concluded that discontinuation of EAT after 3 days in febrile neutropenia is safe in a subset of hemodynamically stable patients without positive blood cultures. However, no control group was available in this study (10).

As of 2011, a new protocol promoting early discontinuation of EAT adapted from Slobbe et al. (10), has been introduced at the haematology department of the University Medical Centre Utrecht (UMCU). This offered the opportunity to assess with a historical control group the effect of this protocol on carbapenem and vancomycin use and its safety in AML and high-risk MDS patients with febrile neutropenia.

\section{Methods}

\section{Patient population}

This retrospective before-after study was performed with a cohort of haematological patients treated from 2007-2014 at the haematology department of the UMCU. The intervention consisted of the introduction of a new local protocol that, in contrast to the previous local protocol, promoted early discontinuation of EAT with carbapenems and discouraged standard empirical treatment with vancomycin. This new protocol was started on January $1^{\text {st }} 2011$.

All adult patients ( $\geq 18$ years) with the diagnoses AML or high risk MDS, treated between January $1^{\text {st }}$ 2007 and December $31^{\text {st }} 2014$ in the UMCU with at least one period of prolonged and profound neutropenia were included. Of these patients, only neutropenic episodes related to intensive chemotherapy (including one of the following cytostatic agents: idarubicin, cytarabine, daunorubicin, vincristine, adriamycin, mitoxantrone or etoposide) or allogenic SCT were included. Neutropenic episodes following other chemotherapeutic regimens or unrelated to chemotherapy or allogenic SCT were 
excluded. Neutropenic episodes between January $1^{\text {st }} 2007$ and October $1^{\text {st }} 2010$ (period I) were compared to neutropenic episodes between April $1^{\text {st }} 2011$ and December $31^{\text {st }} 2014$ (period II, after starting the new protocol). The period from October $1^{\text {st }} 2010$ to April $1^{\text {st }} 2011$ was considered a transition period, therefore neutropenic episodes occurring within this interval were excluded. Complete patient data was not available before 2007 , therefore the maximal period that could be studied before the intervention was 45 months (taken into account the transition period). A similar length was chosen for study period II, i.e. 45 months, making the total study period eight years (2007-2014).

\section{Definition of profound and prolonged neutropenia}

Neutropenia was defined as at least two consecutive neutrophil measurements of $<0.5 \times 10^{9} \mathrm{cell} / \mathrm{s} / \mathrm{L}$ within 90 days. A single neutrophil count above $0.5 \times 10^{9}$ cells/L was ignored, if flanked by neutrophil counts below $<0.5 \times 10^{9}$ cells $/ L$ within one week. Only prolonged neutropenic episodes, with a duration of seven days or more, occurring within fourteen days after start of chemotherapy or conditioning for allogenic SCT were taken into account. These neutropenic episodes are frequently accompanied by mucositis. If chemotherapy or allogenic SCT was started during a period of pre-existing neutropenia, the ensuing neutropenia was considered to be (at least partially) treatment- related if the neutropenia continued for at least seven days after start of treatment.

Prophylaxis and antimicrobial treatment; old protocol

\section{Data sources}

A primary database containing patient data extracted from hospital electronic patient record systems was set up using the Research Data Platform in the UMCU. This database consisted of all patients linked to the diagnosis or treatment of AML or MDS. Data of prescribed antibacterial therapy and cell-count data used to identify neutropenic episodes, were derived from the Utrecht Patient Oriented Database (UPOD) (12). Data of administered cytostatic agents and data concerning allogenic SCT in our patient selection was collected from the in-hospital pharmacy department of the UMCU and the treatment files of the haematology department, respectively. Data of positive blood cultures was derived from the General Laboratory Information Management System (GLIMS). This study was performed in accordance with the ethical standards of our centre.

\section{Outcome measurements}

The primary outcome was carbapenem and vancomycin use within neutropenia following chemotherapy or conditioning for allogenic SCT in AML and high-risk MDS patients. Because neutropenia following chemotherapy or SCT was considered as the period that patients were most at risk for severe infections and the intervention involved a policy change regarding antibiotic use within this period of neutropenia, antibiotic use was expressed as total days of therapy (DOT) per 100 neutropenic days. Interrupted time series (ITS) analysis was performed to assess pre-existing trends, the immediate effect of intervention (step change) and the sustainability of this effect in period II. In addition, the overall use of carbapenems 
and vancomycin within period I, defined as the sum of DOT divided by the sum of neutropenic day, was compared to the overall use in period II.

As secondary outcome the use of other antibiotic agents was analysed, expressed as DOT per 100 neutropenic days. Total antibiotic use consisted of the sum of DOT of all antibiotics combined. If a patient used 2 (or more) different antibiotics on a particular day this was counted as 2 (or more) DOT. Cotrimoxazole was analysed when dosed in 960 mg BID, leaving out Pneumocystis jirovecii/ Toxoplasma prophylaxis after allogenic SCT (480 mg QD).

Other secondary outcomes were 30-day mortality, ICU-admission within 30 days after start EAT and blood cultures positive for microorganisms sensitive to imipenem. These outcomes were measured for the neutropenic episodes in which EAT was started. Cases of mortality within 30 days after start of EAT with a carbapenem were reviewed separately by three of the authors (AN, AB and TVDB). Overall mortality, infection-associated mortality and carbapenem preventable mortality were distinguished. Infectionassociated mortality was defined as clinical signs and/or microbiologically results compatible with infection at time of death. Carbapenem preventable mortality was defined as infection-associated mortality where continuing EAT possibly could have prevented the adverse outcome, because of a suspected or proven etiologic agent that was carbapenem sensitive.

Positive blood cultures within neutropenia drawn after discontinuation of EAT were analysed, because the goal was to study the possible adverse consequences of early discontinuation of carbapenems (e.g. infection/bacteraemia with carbapenem sensitive microorganisms).

\section{Data analysis}

Analysis was conducted at the level of the neutropenic episodes. Changes in carbapenem use and vancomycin use were analysed by ITS. Effect of intervention was represented by a step change (representing the immediate effect). A segmented linear mixed regression model was used with a random intercept for individual patients to correct for multiple neutropenic episodes per patient. Analysis was not corrected for confounders. The $95 \%$ confidence interval was derived through bootstrapping with 20000 iterations. For all antibiotics included in this study, the overall use of different antibiotics within period I was compared to period II, expressed in DOT/100 neutropenic days. Data-analysis was performed using SAS enterprise Guide 7.1. Statistical analysis was done using R version 3.5.1.

\section{Results}

\section{Patients and neutropenic episodes}

In total, 234 patients with AML or MDS and neutropenia were identified with a total number of 494 neutropenic episodes. After exclusion of episodes not related to chemotherapy or allogenic SCT and 20 neutropenic episodes during the transition period, 362 neutropenic episodes remained for further analysis (figure 2). These neutropenic episodes involved 201 individual patients, of which 184 were diagnosed 
with AML and 17 with high-risk MDS (table 1). In AML patients 267 neutropenic episodes were associated with intensive chemotherapy and 72 neutropenic episodes were associated with allogenic SCT (not shown). Further analysis of neutropenic episodes, primary and secondary outcomes, was performed on neutropenic episodes with AML and high-risk MDS patients grouped together. Characteristics of the neutropenic episodes are shown in table 2. The median duration of neutropenia of all neutropenic episodes combined was 18 days in both period I (IQR 12-25) and period II (IQR 12-26). (table 2)

\section{Primary outcome}

ITS analysis showed a significant step change in carbapenem use of 16.1 DOT/100 neutropenic days $(95 \% \mathrm{Cl}-26.73$ to -1.41$)$ as shown in figure 3 . In both periods there had been upward trends in carbapenem use but these were not significant. Overall carbapenem use within period I was 40.3 DOT/100 neutropenic days versus 31.6 DOT/100 neutropenic days in period II, a decrease of $21.6 \%$ (table 3). Vancomycin use decreased significantly after intervention with a step change of 13.7 DOT/100 neutropenic days ( $95 \% \mathrm{Cl}-23.66$ to -2.90 ) (figure 4). In both period I and period II there had been a downward trend in vancomycin use, though not significant. Overall vancomycin use decreased from 26.7 DOT/100 neutropenic days in period I to 12.1 DOT/100 neutropenic days in period II, a decrease of $54.7 \%$ (table 3).

\section{Secondary outcomes}

The overall use of several antibiotics in period I and within period II is shown in table 3 . The results show no major differences in the use of other therapeutically used antibiotic agents. The total use of prophylactic antibiotics in period I (83.9 DOT/100 neutropenic days) was slightly higher in period II (87.7 DOT/100 neutropenic days). Total antibiotic use decreased from 155.6 DOT/100 neutropenic days in period I to 138.0 DOT/100 neutropenic days in period II, a reduction of $11.3 \%$ (table 3 ).

No apparent difference in mortality associated with an infection that could have been prevented with continued carbapenem treatment was identified. The overall mortality within 30 days after starting with EAT was 5.6\% (in 15/268 neutropenic episodes). Eleven fatal cases were associated with infection: two in period I and nine in period II. However, these cases were all classified as unlikely to be preventable with continuation of carbapenem therapy for the following reasons. Firstly, a carbapenem was actually continued $(n=5)$, according to the protocol in hemodynamically instable patients. Secondly, the pathogen was not susceptible to carbapenems $(n=3)$, i.e. mucormycosis, pulmonary aspergillosis and invasive Candidiasis with Candida glabrata. Thirdly, appropriate targeted therapy was given, based on microbiological findings $(n=1)$, i.e. a case of Clostridium difficile pseudomembranous colitis in combination with Stenotrophomonas maltophilia bacteraemia, treated with metronidazole and levofloxacin. Finally, presumed appropriate targeted therapy was given based on a clinical diagnosis $(n=2)$, i.e. a patient with cellulitis treated with cefuroxim and a patient with neutropenic enterocolitis treated with a carbapenem which was interrupted for one day. Twelve days after interruption of EAT this last patient died of a bleeding in the liver, which was considered not to be related to colitis. For all cases in which EAT was discontinued, ciprofloxacin prophylaxis was administered according to the protocol. 
No notable difference between the number of ICU-admissions within 30 days after starting EAT was observed, 9/116 neutropenic episodes in period I (7.8\%) and 9/152 neutropenic episodes in period II (5.9\%).

During the study period, blood cultures obtained after discontinuation of EAT were positive in 56 of 362 neutropenic episodes (15\%). In six of these neutropenic episodes blood cultures were positive for microorganisms sensitive to imipenem, i.e. Streptococcus mitis $(n=1)$, Escherichia coli $(n=1)$, Clostridium perfringens $(n=1)$, Pseudomonas aeruginosa $(n=1)$ and Enterococcus spp. $(n=2)$. Four of these positive blood cultures were obtained in period I and two in period II. One blood culture in period II was positive for Enterococcus spp. and one for Pseudomonas aeruginosa. In all cases adequate therapy was started when blood culture results became available. No fatal outcomes were recorded within 30 days after early discontinuation of EAT. More details are shown in table 4.

\section{Discussion}

This study shows a reduction in carbapenem and vancomycin use after introduction of a protocol promoting early discontinuation of EAT in high risk haematology patients with febrile neutropenia. According to this protocol, EAT is discontinued after three days (with bacterial prophylaxis restarted), regardless of fever, provided blood cultures remain negative, patients are hemodynamically stable and do not have a pulmonary infection with unknown etiology. Also the standard use of vancomycin as EAT is discouraged in the new protocol, following the IDSA guideline (1). Importantly, both carbapenems and vancomycin are classified by the WHO as key targets of antimicrobial stewardship programs (7).

In period II, carbapenems were started slightly more often compared to period I ( $76 \%$ vs. $72 \%$, table 2$)$. Despite the increased frequency of starting carbapenems, ITS showed a significant reduction (step change, figure 3 ) and the overall use was decreased by $21.6 \%$ (table 3 ). In addition to the reduction of EAT with carbapenems, there was a $54.7 \%$ reduction of vancomycin use. Besides, the effect of intervention on carbapenem and vancomycin use seemed to be sustained over period II.

Analysis of administration of other therapeutically used antibiotics did not show striking increases, indicating that the restrictive use of carbapenems and vancomycin was not compensated by replacement with other broad-spectrum antibiotics. There was a modest increase in the use of prophylactic antibiotics after introduction of the protocol. This can be attributed to theNonetheless, total antibiotic use (prophylactic and therapeutically used agents combined) decreased with $11.3 \%$.

The reduction of EAT after protocol implementation did not lead to an increase in adverse events. Although all-cause mortality was higher in period II, detailed analysis did not reveal causality between early discontinuation of EAT and fatal outcomes. Secondly, there was no notable difference in ICU admittance within 30 days after discontinuing EAT between the two periods. Thirdly, in both periods there were some episodes of bacteraemia with a carbapenem-susceptible microorganism within 30 days after stopping EAT. However, all these episodes of bacteraemia were treated adequately and no mortality within 30 days after early discontinuation of EAT was observed in these cases. 
Shortening the course of EAT in haematological patients with febrile neutropenia remains a subject of debate $(13,14)$ while there is increasing evidence supporting a shorter course of EAT in these high risk haematology patients (8-10). Many of these studies focus on the safety of the Fourth European Conference on Infections in Leukaemia (ECIL-4) recommendation to discontinue EAT after 48 hours of apyrexia $(3,8,9,15)$. This policy reduces EAT in comparison to previous recommendations $(8,9,15)$. However, broad-spectrum antibiotic use may be reduced even further, because febrile neutropenia is frequently unrelated to infection (4-6). In this respect, the study of Slobbe et al. is interesting, describing a protocol to discontinue EAT after three days, regardless of persisting fever (10). The authors did not document any mortality related to an untreated bacterial infection and concluded early discontinuation of EAT is safe, although the study lacked a control group. We compared mortality and other measures of safety before and after implementation of a similar protocol, thereby providing a historical control group.

This study has limitations. Most important, the retrospective design of this before-after study makes it impossible to take into account all time-related factors that could have influenced the outcome measures. Therefore, the results can only be interpreted as descriptive. Two potential time-related factors, i.e. changes in patient population and treatment intensity, may influence the outcomes. More vulnerable patients (e.g. more serious comorbidities) have become eligible for haematological treatment regimens and conditioning regimens for allogenic SCT have become more intense. This may have led to more severe morbidity in the second (intervention) cohort. Despite these changes, potentially leading to an overestimation of adverse events in period II, this study shows a decrease in consumption of EAT and no increased infection-associated morbidity and mortality. However, the frequency of the studied adverse events (e.g. mortality, IC admission and positive blood cultures with imipenem sensitive microorganisms) is relatively small, so the study may be underpowered for the safety outcomes. No data on temperature was available. However, patients were considered febrile when carbapenems were started because febrile neutropenia was an almost exclusive indication for administering carbapenems.

\section{Conclusion}

In conclusion, this study shows that introducing a protocol advocating early discontinuation of EAT in AML and high-risk MDS patients with febrile neutropenia during chemotherapy or conditioning for allogenic SCT, resulted in decreased use of carbapenems and vancomycin, without apparently compromising patient safety. In addition to current recommendations, early discontinuation of EAT without the explicit need for apyrexia is worth further exploration in stable haematological patients with febrile neutropenia.

\section{Declarations}

Ethics approval and consent to participate

This study was performed in accordance with the ethical standards of our centre and approved by the ethics committee medical research (METC Utrecht). 
Consent for publication

Not applicable

Availability of data and materials

The datasets generated and analysed during the current study are not publicly available due to confidentiality, but are available from the corresponding author on reasonable request for researchers who meet the criteria for access.

\section{Funding}

Not applicable

\section{Author's contribution}

T designed and supervised the study, MS developed the methodology and made substantial contributions to the design of the work, MC provided data, FA analysed the data, FA, T, AH and RA interpreted patient data, T and FA wrote the manuscript, the manuscript was revised by all authors. All authors have approved the submitted version.

\section{Acknowledgements}

We thank Pieter-Jan Haas, Francis Verweij, Julia Velikopolskaia, Henri van Werkhoven, Danny Broekhuizen for providing, clinical, laboratory and pharmacy data and valuable help with SAS Enterprise and statistical analysis.

\section{Competing interests}

This research received no specific grant from any funding agency in the public, commercial, or not-forprofit sectors. The authors declare that they have no competing interests.

\section{References}

1. Freifeld A, Bow E, Sepkowitz K, Boeckh M, Ito J, Mullen C, et al. Clinical practice guideline for the use of antimicrobial agents in neutropenic patients with cancer: 2010 update by the infectious diseases society of america. Clin Infect Dis. 2011;52(4):e93.

2. Gafter-Gvili A, Fraser A, Paul M, Leibovici L. Meta-analysis: antibiotic prophylaxis reduces mortality in neutropenic patients. Ann Intern Med [Internet]. 2005;142(12 Pt 1):979-95. Available from: http://www.ncbi.nlm.nih.gov/pubmed/15968013 
3. Averbuch D, Orasch C, Cordonnier C, Livermore D, Mikulska M, Viscoli C, et al. European guidelines for empirical antibacterial therapy for febrile neutropenic patients in the era of growing resistance: summary of the 2011 4th European Conference on Infections in Leukemia. Haematologica. 2013;98(12):1826-35.

4. Pagano L, Caira M, Nosari A, Rossi G, Viale P, Aversa F, et al. Etiology of febrile episodes in patients with acute myeloid leukemia: results from the Hema e-Chart Registry. Vol. 171, Archives of internal medicine. United States; 2011. p. 1502-3.

5. van der Velden WJFM, Herbers AHE, Netea M, Blijlevens NMA. Mucosal barrier injury, fever and infection in neutropenic patients with cancer: introducing the paradigm febrile mucositis. $\mathrm{Br} \mathrm{J}$ Haematol. 2014;167(4):441-52.

6. Wenneras C, Hagberg L, Andersson R, Hynsjo L, Lindahl A, Okroj M, et al. Distinct inflammatory mediator patterns characterize infectious and sterile systemic inflammation in febrile neutropenic hematology patients. PLoS One. 2014;9(3):e92319.

7. Sharland M, Pulcini C, Harbarth S, Zeng M, Gandra S, Mathur S, et al. Classifying antibiotics in the WHO Essential Medicines List for optimal use-be AWaRe. Lancet Infect Dis. 2018 Jan;18(1):18-20.

8. Clech L Le, Talarmin J-P, Couturier M-A, lanotto J-C, Nicol C, Calloch R Le, et al. Early discontinuation of empirical antibacterial therapy in febrile neutropenia: the ANTIBIOSTOP study. Infect Dis (Auckl). 2018;50(7):539-49.

9. Guisado MA, Espigado I, Peña AM, Gudiol C, Cebrecos CR, Falantes J, et al. Optimisation of empirical antimicrobial therapy in patients with haematological malignancies and febrile neutropenia (How Long study): an open-label, randomised, controlled phase 4 trial. Lancet Haematol. 2017;4(12):e583.

10. Slobbe L, van der Waal L, Jongman LR, Lugtenburg PJ, Rijnders BJ. Three-day treatment with imipenem for unexplained fever during prolonged neutropaenia in haematology patients receiving fluoroquinolone and fluconazole prophylaxis: A prospective observational safety study. Eur $\mathrm{J}$ Cancer. 2009;45(16):2810-7.

11. Norrby SR. Neurotoxicity of carbapenem antibacterials. Drug Saf. 1996 Aug;15(2):87-90.

12. ten Berg MJ, Huisman A, van den Bemt PMLA, Schobben AFAM, Egberts ACG, van Solinge WW. Linking laboratory and medication data: new opportunities for pharmacoepidemiological research. Clin Chem Lab Med. 2007;45(1):13-9.

13. Micol J-B, Chahine C, Woerther P-L, Ghez D, Netzer F, Dufour C, et al. Discontinuation of empirical antibiotic therapy in neutropenic acute myeloid leukaemia patients with fever of unknown origin: is it ethical? Clin Microbiol Infect. 2014 Jul;20(7):0453-5.

14. Orasch C, Averbuch D, Mikulska M, Cordonnier C, Livermore DM, Gyssens IC, et al. Discontinuation of empirical antibiotic therapy in neutropenic leukaemia patients with fever of unknown origin is ethical. Vol. 21, Clinical microbiology and infection : the official publication of the European Society of Clinical Microbiology and Infectious Diseases. England; 2015. p. e25-7.I

15. la Martire G, Robin C, Oubaya N, Lepeule R, Beckerich F, Leclerc M, et al. De-escalation and discontinuation strategies in high-risk neutropenic patients: an interrupted time series analyses of 
antimicrobial consumption and impact on outcome. Eur J Clin Microbiol Infect Dis. 2018

Oct;37(10):1931-40.

\section{Tables}

\section{Table 1: Patient characteristics}

\begin{tabular}{rrll}
\hline & & Period I & Period II \\
\hline Total & $\mathrm{n}$ & 97 & 104 \\
Diagnosis & AML & $90(93 \%)$ & $94(90 \%)$ \\
& High-risk MDS & $7(7 \%)$ & $10(10 \%)$ \\
Sex & Male & $46(47 \%)$ & $48(46 \%)$ \\
Age & Years \pm SD & $50.7 \pm 14.7$ & $53.4 \pm 15.1$ \\
\hline
\end{tabular}

Abbreviations: AML, acute myeloid leukaemia; MDS, myelodysplastic syndrome

\section{Table 2: Characteristics of neutropenic episodes}

\begin{tabular}{|c|c|c|c|c|c|c|c|c|c|}
\hline & \multicolumn{4}{|c|}{ Neutropenic episodes in period I } & \multicolumn{5}{|c|}{ Neutropenic episodes in period II } \\
\hline & \multirow{2}{*}{$\begin{array}{l}\text { Total } \\
n\end{array}$} & \multirow{2}{*}{$\begin{array}{l}\text { Duration in } \\
\text { days } \\
\text { median (IQR) }\end{array}$} & \multicolumn{2}{|c|}{$\begin{array}{l}\text { Carbapenem } \\
\text { started }\end{array}$} & \multirow{2}{*}{$\begin{array}{l}\text { Total } \\
n \\
\end{array}$} & \multirow{2}{*}{\multicolumn{2}{|c|}{$\begin{array}{l}\text { Duration in } \\
\text { days } \\
\text { median }(I Q R)\end{array}$}} & \multicolumn{2}{|c|}{$\begin{array}{l}\text { Carbapenem } \\
\text { started }\end{array}$} \\
\hline & & & $n$ & $\%$ & & & & $n$ & $\%$ \\
\hline Chemotherapy & 128 & $19 \quad(14-26)$ & 105 & $(82)$ & 153 & 18 & $(13-25)$ & 123 & $(80)$ \\
\hline Allogenic SCT & 34 & $(8-17)$ & 11 & $(32)$ & 47 & 12 & $(9-26)$ & 29 & $(62)$ \\
\hline Total & 162 & $(12-25)$ & 116 & $(72)$ & 200 & 18 & $(12-26)$ & 152 & (76) \\
\hline
\end{tabular}




\begin{tabular}{llll}
\hline & & \multicolumn{2}{l}{ Days of therapy/100 neutropenic days } \\
& & Period I & Period II \\
\hline Therapeutical agents & Carbapenems & 40.3 & 31.6 \\
& Vancomycin & 26.7 & 12.1 \\
\cline { 2 - 4 } & Ceftazidime & 0.1 & 0.3 \\
\cline { 2 - 4 } & Ceftriaxone & 0.8 & 0.8 \\
\cline { 2 - 4 } Prophylactic agents & Piperacillin/tazobactam & 0.2 & 1.1 \\
\cline { 2 - 4 } & Penicillin & 3.1 & 3.8 \\
\cline { 2 - 4 } & Aminoglycosides & 0.5 & 0.6 \\
\cline { 2 - 4 } Total & Clindamycin & 10.9 & 7.0 \\
\cline { 2 - 4 } & Ciprofloxacin & 65.8 & 60.1 \\
\cline { 2 - 4 } & Cotrimoxazole $*$ & 3.1 & 5.6 \\
\cline { 2 - 4 } & Cefazolin & 4.1 & 15.0 \\
\hline \multirow{2}{*}{ Tose } & & 155.6 & 138.0 \\
\hline
\end{tabular}

*dose > $960 \mathrm{mg} B I D$

Table 4: Positive blood cultures with carbapenem sensitive microorganisms within 30 days after discontinuation of EAT

\begin{tabular}{|c|c|c|c|c|c|c|}
\hline & $\begin{array}{l}\text { Patient } \\
\text { (sex, age, } \\
\text { diagnosis) }\end{array}$ & $\begin{array}{l}\text { Duration } \\
\text { of initial } \\
\text { EAT }\end{array}$ & $\begin{array}{l}\text { Micro-organism in } \\
\text { blood culture }\end{array}$ & $\begin{array}{l}\text { Days between } \\
\text { discontinuation } \\
\text { of EAT and } \\
\text { positive blood } \\
\text { culture }\end{array}$ & $\begin{array}{l}\text { Focus of } \\
\text { infection }\end{array}$ & Treatment \\
\hline \multirow[t]{4}{*}{$\begin{array}{l}\text { Period } \\
\text { I }\end{array}$} & $\begin{array}{l}\text { F 51, } \\
\text { AML }\end{array}$ & 11 & Enterococcus species & 4 & $\begin{array}{l}\text { Central } \\
\text { venous } \\
\text { catheter }\end{array}$ & $\begin{array}{l}\text { Vancomycin, followed } \\
\text { by amoxicillin }\end{array}$ \\
\hline & $\begin{array}{l}\text { F } 44 \\
\text { AML }\end{array}$ & 7 & Streptococcus mitis & 6 & Sinusitis & $\begin{array}{l}\text { Restart EAT with a } \\
\text { carbapenem }\end{array}$ \\
\hline & $\begin{array}{l}\text { F 65, } \\
\text { MDS }\end{array}$ & 7 & Escherichia coli & 11 & Urosepsis & $\begin{array}{l}\text { Restart EAT with a } \\
\text { carbapenem, followed } \\
\text { by ceftriaxone }\end{array}$ \\
\hline & $\begin{array}{l}\text { M 59, } \\
\text { AML }\end{array}$ & 16 & $\begin{array}{l}\text { Clostridium } \\
\text { perfringens }\end{array}$ & 7 & $\begin{array}{l}\text { Translocation } \\
\text { of infected } \\
\text { trombus }\end{array}$ & $\begin{array}{l}\text { Restart EAT with a } \\
\text { carbapenem }+ \\
\text { vancomycin }\end{array}$ \\
\hline \multirow[t]{2}{*}{$\begin{array}{l}\text { Period } \\
\text { II }\end{array}$} & $\begin{array}{l}\text { M 49, } \\
\text { AML }\end{array}$ & 4 & $\begin{array}{l}\text { Pseudomonas } \\
\text { aeruginosa }\end{array}$ & 6 & Dental focus & $\begin{array}{l}\text { Piperacillin/tazobactam } \\
+ \text { tobramycin }\end{array}$ \\
\hline & $\begin{array}{l}\text { M 42, } \\
\text { AML }\end{array}$ & 5 & Enterococcus species & 17 & Unknown & Vancomycin \\
\hline
\end{tabular}

Abbreviations: F, female; M, male; AML, acute myeloid leukaemia; MDS, myelodysplastic syndrome; EAT, empirical antibiotic treatment

\section{Figures}




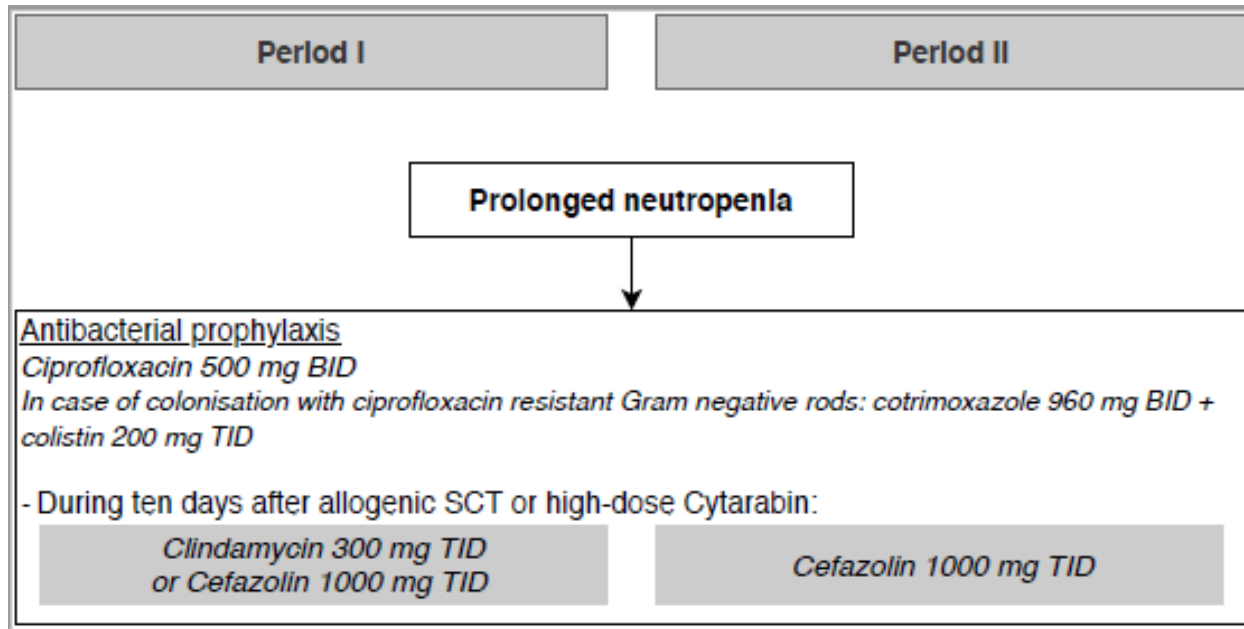

\section{Antifungal prophylaxis}

Fluconazole $150 \mathrm{mg}$ QD

- In case of colonisation with C. krusei or C. glabrata:

\section{Amphotericin B deoxycholate $200 \mathrm{mg}$ TID No fungal prophylaxis}

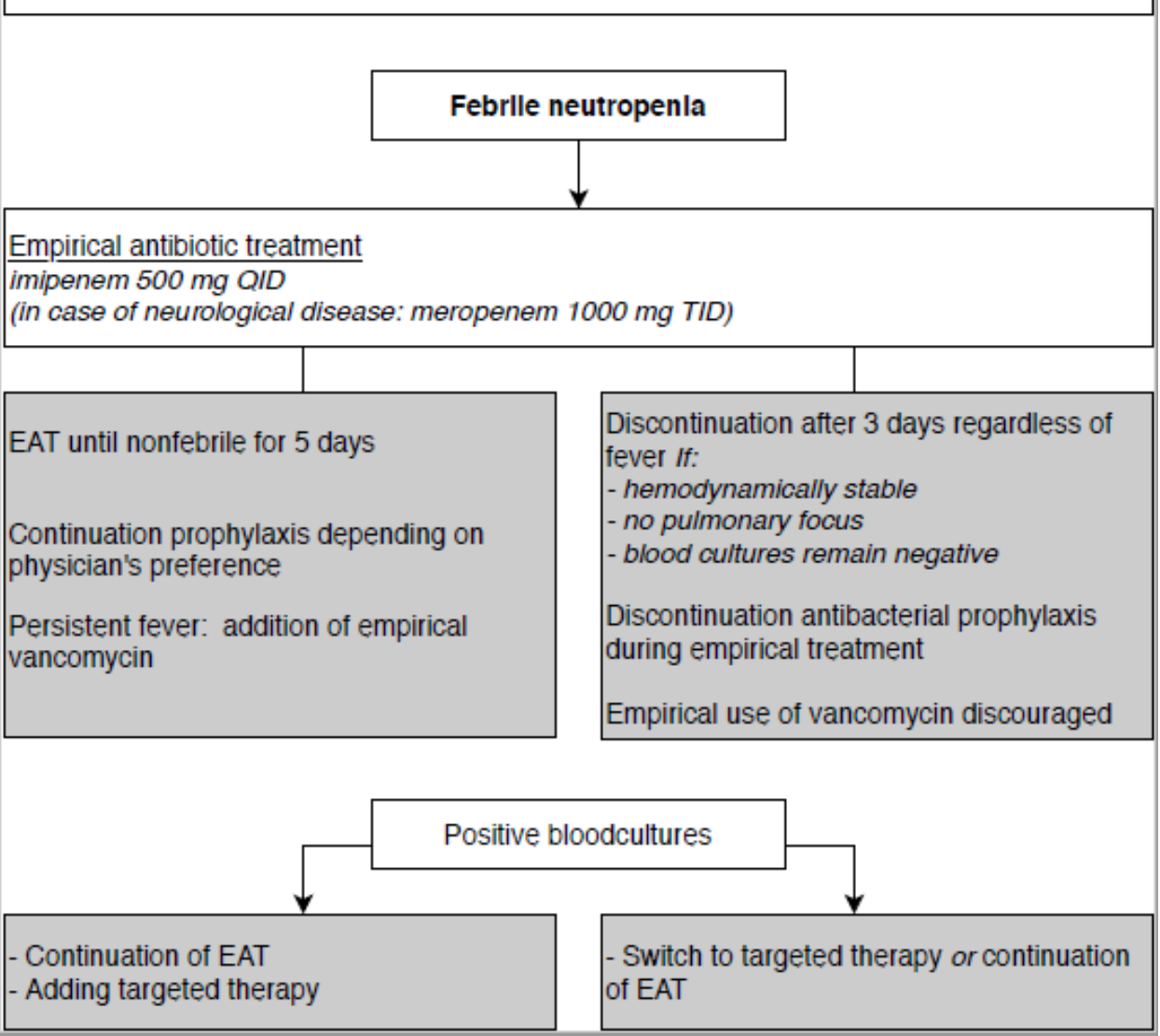

\section{Figure 1}

Prophylaxis and antibiotic treatment protocol 


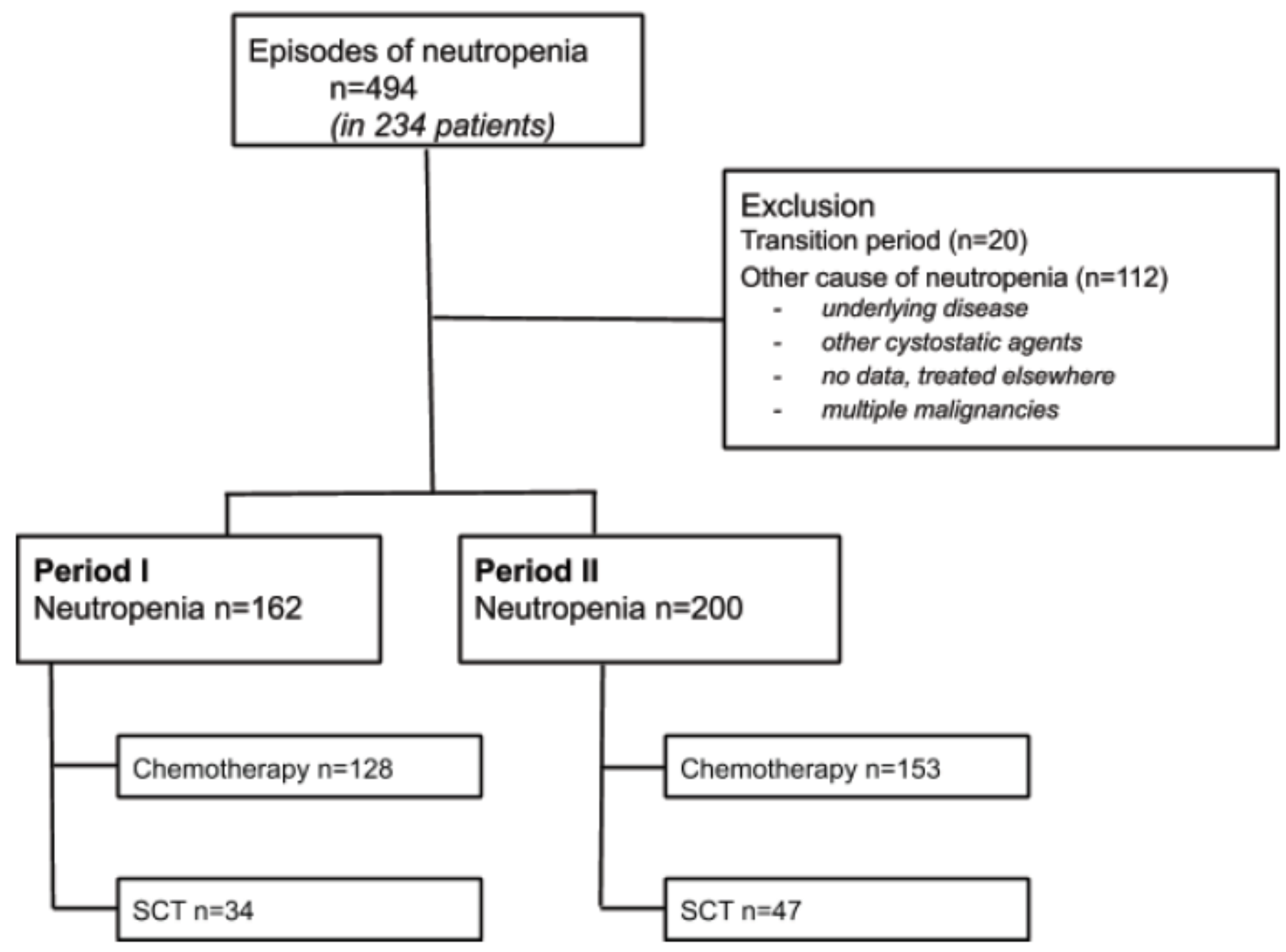

Figure 2

Selection of neutropenic episodes 


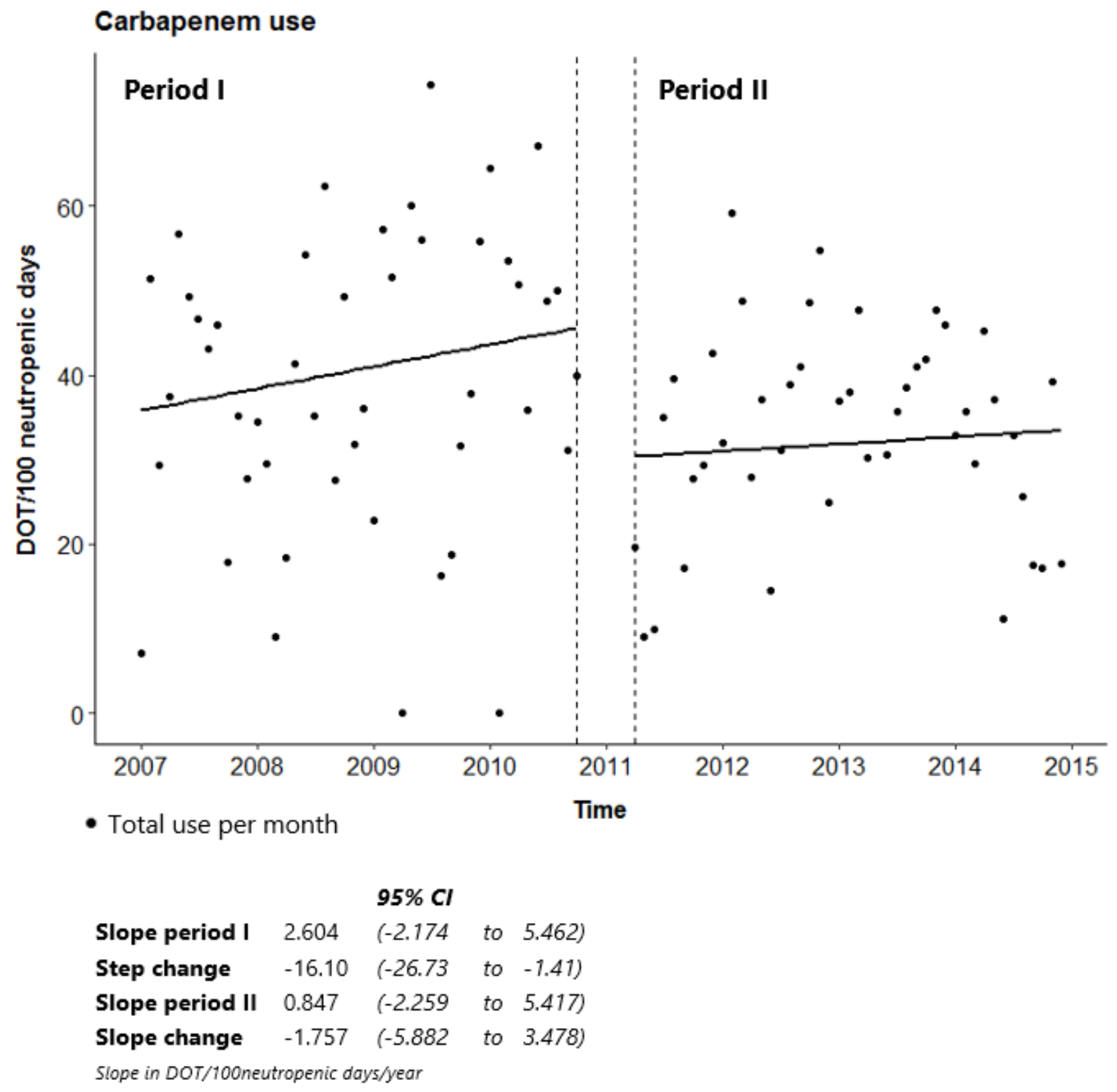

Figure 3

ITS analysis of carbapenem use 


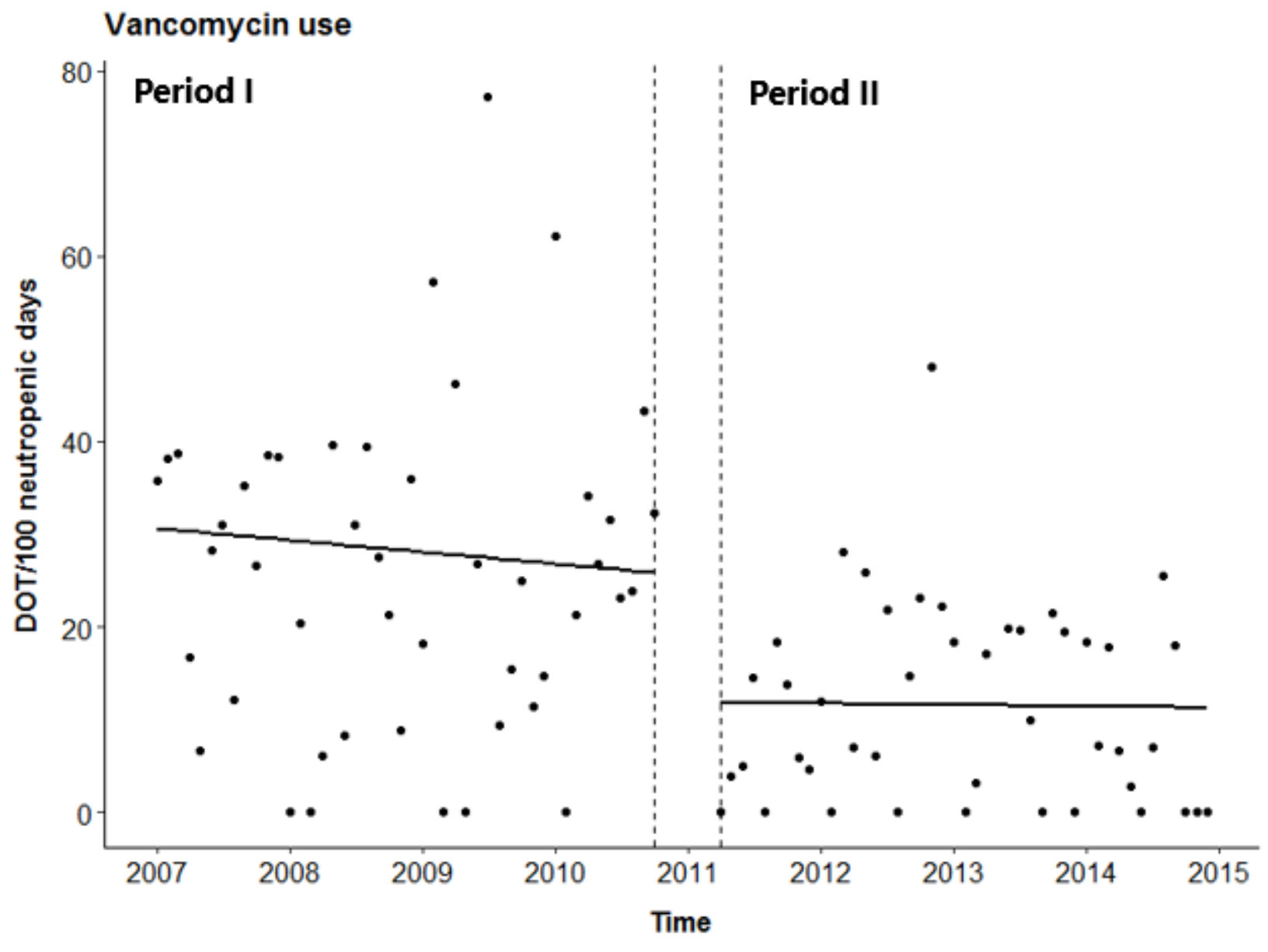

- Total use per month

$95 \% \mathrm{Cl}$

Slope period I $-1.275 \quad(-5.450$ to 2.194)

Step change $-13.67 \quad(-23.66$ to -2.90$)$

Slope period II $-0.146 \quad(-0.215$ to 5.469$)$

Slope change $1.129 \quad(-2.555$ to 6.205$)$

Slope in DOT/100neutropenic days/year

Figure 4

ITS analysis of vancomycin use

\section{Supplementary Files}

This is a list of supplementary files associated with this preprint. Click to download.

- SupplementaryFile.pdf 